\title{
INTRINSIC CIRCLE DOMAINS
}

\author{
EDWARD CRANE
}

\begin{abstract}
Using quasiconformal mappings, we prove that any Riemann surface of finite connectivity and finite genus is conformally equivalent to an intrinsic circle domain $\Omega$ in a compact Riemann surface $S$. This means that each connected component $B$ of $S \backslash \Omega$ is either a point or a closed geometric disc with respect to the complete constant curvature conformal metric of the Riemann surface $(\Omega \cup B)$. Moreover, the pair $(\Omega, S)$ is unique up to conformal isomorphisms. We give a generalization to countably infinite connectivity. Finally, we show how one can compute numerical approximations to intrinsic circle domains using circle packings and conformal welding.
\end{abstract}

\section{INTRODUCTION}

Let $\Omega$ be a finitely connected domain in the Riemann sphere $\widehat{\mathbb{C}}$. A classical theorem of Koebe states that $\Omega$ is conformally equivalent to the complement of a finite set of pairwise disjoint closed discs and points. Such a domain is called a finitely connected circle domain. Koebe's theorem was extended by $\mathrm{He}$ and Schramm [5] to apply to domains with countably many complementary components. Schramm later gave a different proof of this result using transboundary extremal length [8].

Theorem A ([5, Theorem 0.1]). Let $\Omega$ be a domain in $\widehat{\mathbb{C}}$ such that boundary $\partial \Omega$ has at most countably many components. Then $\Omega$ is conformally homeomorphic to a circle domain $\Omega^{*}$ in $\widehat{\mathbb{C}}$. Moreover, $\Omega^{*}$ is unique up to Möbius transformations and every conformal automorphism of $\Omega^{*}$ is the restriction of a Möbius transformation.

If $\Omega$ is a domain in $\widehat{\mathbb{C}}$ that has at least three complementary components, or at least one complementary component that is not a puncture, then we say that $\Omega$ is hyperbolic. The reason is that there is then an unbranched analytic covering map from the unit disc $\mathbb{D}$ onto $\Omega$, which can be used to transfer the Poincaré metric on $\mathbb{D}$ to a complete conformal metric of constant curvature -1 on $\Omega$, which is called the hyperbolic metric.

Every ring domain is conformally equivalent to a round annulus, a punctured disc, or the punctured plane. For a round annulus $\widehat{\mathbb{C}} \backslash\left(B_{1} \cup B_{2}\right)$, each complementary component $B_{i}$ is a spherical disc, i.e., a closed ball in the spherical metric. Observe that $B_{i}$ is also a closed ball with respect to the hyperbolic metric belonging to the domain $\Omega \cup B_{i}$, which is a larger spherical disc. In this paper we generalize this property to obtain a new canonical form for multiply connected domains.

Received by the editors March 27, 2013.

2010 Mathematics Subject Classification. Primary 30C20; Secondary 30F45, 30C30, 52C26.

Key words and phrases. Circle domains, hyperbolic metric, circle packing, conformal welding. 
Theorem 1. Let $\Omega$ be a finitely connected domain in the Riemann sphere $\widehat{\mathbb{C}}$ with complementary components $K_{1}, \ldots, K_{n}$. Suppose that each domain $\Omega \cup K_{i}$ is hyperbolic. Then $\Omega$ is conformally equivalent to a domain $\Omega^{*}$ in $\widehat{\mathbb{C}}$ with complementary components $L_{1}, \ldots, L_{n}$ such that for each $i=1, \ldots, n, \Omega \cup L_{i}$ is hyperbolic and either $L_{i}$ is a puncture or $L_{i}$ is a closed disc with respect to the hyperbolic metric of $\Omega \cup L_{i}$. Moreover, $\Omega^{*}$ is unique up to Möbius transformations.

We call the canonical domains given by this theorem intrinsic circle domains.

Theorem 1 has a generalization to positive genus ambient surfaces in place of $\widehat{\mathbb{C}}$, which we will now explain. In any Riemann surface $S$, a closed geometric disc will mean a closed ball of some positive radius with respect to the appropriate complete conformal metric of constant curvature on $S$, (i.e., hyperbolic, Euclidean or spherical), with the extra condition that it must be homeomorphic to the closed unit disc. Equivalently, the radius of the ball must be strictly less than the injectivity radius of the metric at the center of the disc. A circle domain $\Omega^{*}$ in $S$ is a connected open subset of $S$ for which each complementary component is either a point or a closed geometric disc in $S$. Via the uniformization theorem, He and Schramm extended Theorem $\mathrm{A}$ to this setting, as follows.

Theorem B ([5, Theorem 0.2]). Let $\Omega$ be an open Riemann surface with finite genus and at most countably many ends. Then there is a closed Riemann surface $R^{*}$ such that $\Omega$ is conformally homeomorphic to a circle domain $\Omega^{*}$ in $R^{*}$. Moreover, the pair $\left(R^{*}, \Omega^{*}\right)$ is unique up to conformal homeomorphisms.

Likewise, we can extend Theorem 1 to deal with the case of arbitrary finite genus. For the moment we also restrict ourselves to finite connectivity; we will relax this condition in $\$ 5$,

Theorem 2. Let $\Omega$ be a Riemann surface of finite genus and finite connectivity. Then there is a conformal embedding $\varphi$ of $\Omega$ into a compact Riemann surface $S$ of the same genus as $\Omega$ so that $S \backslash \varphi(\Omega)$ is the union of disjoint closed, connected and simply connected sets $L_{1}, \ldots, L_{n}$, and for each $i \in\{1, \ldots, n\}, L_{i}$ is either a single point or a closed geometric disc with respect to the Riemann surface $\varphi(\Omega) \cup L_{i}$. Moreover, the pair $(S, \varphi)$ is unique up to conformal homeomorphisms.

The condition that $\Omega$ and $S$ have the same genus means that $S$ has the minimal possible genus among all compact Riemann surfaces into which $\Omega$ may be embedded. We will refer to the domain $\varphi(\Omega)$ as an intrinsic circle domain in $S$. Note that Theorem 2 includes Theorem 1 as the special case of genus 0 , so this terminology is consistent; we shall only give a proof of Theorem 2 .

There are some simple special cases. For example, there is precisely one case in which the appropriate geometry of $\varphi(\Omega) \cup L_{i}$ is spherical; this occurs when $\Omega$ is simply connected, in which case the statement reduces to the Riemann mapping theorem. The doubly connected genus 0 cases occur when $\Omega$ is conformally equivalent to the punctured plane $\mathbb{C}^{*}$, the punctured disc $\mathbb{D}^{*}$, or a round annulus. Note that in the case of the punctured disc, the appropriate geometry for $\varphi(\Omega) \cup L_{i}$ is Euclidean for one complementary component and hyperbolic for the other. Another case in which more than one type of geometry must be considered occurs when $\Omega$ is a simply connected domain with two points removed; then the resulting canonical domain is either a triply-punctured sphere or it is $\mathbb{C}^{*} \backslash K$, where $K$ is the image under the exponential map of a closed disc of radius less than $\pi$. If $\Omega$ is a 
Riemann surface of genus 1 with one end, then $\Omega^{*}$ is the complement of a point or of a Euclidean disc in $\mathbb{C} / \Lambda$, where $\Lambda$ is a lattice in $\mathbb{C}$. In all other cases the natural geometry of $\varphi(\Omega) \cup L_{i}$ is hyperbolic for every complementary component $L_{i}$.

Our definition of intrinsic circle domains was motivated by an observation about extremal cases in the Grötzsch or Pólya-Chebotarev problem, which asks for the minimizer of the logarithmic capacity among compact connected sets containing a given finite set of points in $\mathbb{C}$. This problem often appears in the course of studying other extremal problems in geometric function theory. For example, there are recent applications to the Bloch-Landau constant [2] and to Smale's mean value conjecture [3]. It is a classical result of Lavrentiev and Goluzin that the extremal continuum $E$ is unique and is the union of finitely many analytic $\operatorname{arcs} A_{i}$, which are trajectories of a certain rational quadratic differential (see for example [4]). It is not hard to show that each arc $A_{i}$ is a geodesic arc in the hyperbolic metric of the domain $(\widehat{\mathbb{C}} \backslash E) \cup A_{i}$. A similar condition arises for local minima of the condenser capacity for domains separating one finite set of points from another. In [1, 6,9 it is observed that the extremal continuum $E$ enjoys harmonic symmetry. This means that for any subarc $I$ of $E$, the harmonic measure of the complementary domain $\widehat{\mathbb{C}} \backslash E$ with respect to the point $\infty$ assigns equal masses to each side of $I$. Note that both the geodesic arc condition and the harmonic symmetry condition have the property that they may be verified by checking each arc $A_{i}$ separately. To check $A_{i}$, we need to know only the conformal class of the pair $\left(\widehat{\mathbb{C}} \backslash E, A_{i}\right)$. Our notion of intrinsic circle domains arose by analogy with this property.

We now outline the rest of the paper. Section 2 gives a simple qualitative distortion bound for quasiconformal extensions of conformal maps between ring domains, which we later use several times. Section 3 gives the proof of uniqueness in Theorem 2. and section 4 gives the existence proof. In section 5, we extend Theorem 2 to include some cases with countably infinitely many complementary components, subject to a geometric constraint. Section 6 deals with a mixed condition, in which some boundary components are required to be circles in the spherical metric while the others are required to be intrinsic circles in the sense of Theorem 11. In section 7 we discuss the use of circle packings for the numerical approximation of intrinsic circle domains, and illustrate with some examples.

\section{A GeOMETRIC LEMMA}

Lemma 3. Suppose $A_{1}$ and $A_{2}$ are ring domains in $\mathbb{C}$ whose inner boundaries are circles $C_{1}$ and $C_{2}$ respectively. Suppose there is a conformal homeomorphism $F: A_{1} \rightarrow A_{2}$ under which $C_{1}$ corresponds to $C_{2}$. Then the induced homeomorphism $F: C_{1} \rightarrow C_{2}$ has a $K$-quasiconformal extension between the interior discs of $C_{1}$ and $C_{2}$, where the constant $K$ depends only on the conformal modulus of $A_{1}$.

Proof. Lemma 3 is a consequence of [5, Thm 1.4], but it can also be proved simply as follows. After applying similarities, we may assume that $C_{1}$ and $C_{2}$ are both the unit circle $\partial \mathbb{D}$. Let $m$ be the conformal modulus of $A_{1}$. Apply Schwarz reflection across $\partial \mathbb{D}$ to extend $F$ to a conformal homeomorphism $\tilde{F}$ between ring domains $\tilde{A}_{1}$ and $\tilde{A}_{2}$. If $m=\infty$, then $\tilde{F}$ is a conformal homeomorphism from the punctured plane to itself, so is in fact a similarity, and its restriction to $\partial \mathbb{D}$ is an isometry. Otherwise, the doubled ring domains $\tilde{A}_{i}$ are hyperbolic, $\tilde{F}$ is a hyperbolic isometry, and the hyperbolic length of $\partial \mathbb{D}$ in each domain depends only on $m$. We claim that the density of the hyperbolic metric of $\tilde{A}_{1}$ on $\partial \mathbb{D}$ is bounded above and below in 
terms of $m$. Indeed, the hyperbolic length of $\partial \mathbb{D}$ is a function of $m$, so we get such a bound by applying the Koebe distortion theorem to a single-valued lift of $\log \tilde{F}$ to the universal cover of $\tilde{A}_{1}$.

It follows that $F: \partial \mathbb{D} \rightarrow \partial \mathbb{D}$ is bi-Lipschitz, with constants that depend only on $m$. Therefore the radial interpolation of the boundary correspondence provides a quasiconformal extension with dilatation bounded in terms of the modulus of $A_{1}$.

\section{UNIQUENESS}

Suppose that $\Omega$ and $\Omega^{\prime}$ are two intrinsic circle domains in closed Riemann surfaces $S$ and $S^{\prime}$ respectively. Let $f: \Omega \rightarrow \Omega^{\prime}$ be a conformal homeomorphism. We have to show that $f$ is the restriction of a conformal isomorphism $\tilde{f}: S \rightarrow S^{\prime}$.

First, suppose that $\Omega$ is simply connected or doubly connected. If $\Omega$ is simply connected or is doubly connected with infinite conformal modulus, then $S$ is the Riemann sphere and $\Omega$ is a circle domain. $\Omega^{\prime}$ has the same connectivity as $\Omega$ since they are homeomorphic, so $\Omega^{\prime}$ is also a circle domain in the Riemann sphere $S$. The uniqueness part of Koebe's theorem then shows that $f$ is the restriction of a Möbius map. Otherwise, $\Omega$ is a ring domain of finite modulus, as is $\Omega^{\prime}$. Then $S$ and $S^{\prime}$ are both of genus 0 and we have to show that $f$ extends to a Möbius map. Consider a complementary component $B_{1}$ of $\Omega$, and let $B_{1}^{\prime}$ be the corresponding complementary component of $\Omega^{\prime}$. Let $\pi: \mathbb{D} \rightarrow \Omega \cup B_{1}$ and $\pi^{\prime}: \mathbb{D} \rightarrow \Omega^{\prime} \cup B_{i}^{\prime}$ be any Riemann maps. Then by hypothesis $\pi^{-1}\left(B_{1}\right)$ and $\pi^{\prime-1}\left(B_{1}^{\prime}\right)$ are closed hyperbolic discs in $\mathbb{D}$, which are bounded by Euclidean circles. Thus $\pi^{-1}(\Omega)$ and $\pi^{\prime-1}\left(\Omega^{\prime}\right)$ are round annuli. Any conformal homeomorphism between round annuli is the restriction of a Möbius map, so we can extend $\pi^{\prime-1} \circ f \circ \pi$ to a Möbius map $g$. Now define $f_{1}=\pi^{\prime} \circ g \circ \pi^{-1}$. Then $f_{1}: \Omega \cup B_{1} \rightarrow \Omega^{\prime} \cup B_{1}^{\prime}$ is a conformal homeomorphism extending $f$. Similarly, $f$ extends to a conformal homeomorphism $f_{2}: \Omega \cup B_{2} \rightarrow$ $\Omega \cup B_{2}^{\prime}$. Gluing $f_{1}$ and $f_{2}$ together we obtain a conformal homeomorphism from the Riemann sphere to itself which extends $f$. This must be a Möbius map. Since $\Omega$ is conformally equivalent to some round annulus, which is an intrinsic circle domain, this in fact shows that $\Omega$ is a round annulus.

Now suppose that $\Omega$ is at least triply connected. Consider any complementary component $B_{i}$ of $\Omega$, and let $B_{i}^{\prime}$ be the corresponding complementary component of $\Omega^{\prime}$. This makes sense since the homeomorphism $f$ induces a bijection between the ends of $\Omega$ and the ends of $\Omega^{\prime}$; for an intrinsic circle domain each end corresponds to precisely one complementary component since the complementary components are all contractible.

Since $\Omega$ and $\Omega^{\prime}$ are at least triply connected, $\Omega \cup B_{i}$ and $\Omega^{\prime} \cup B_{i}^{\prime}$ are not simply connected. Therefore we must consider their universal covers, $\mathcal{U}$ and $\mathcal{U}^{\prime}$ respectively, in order to understand their hyperbolic metrics. Let $\pi: \mathcal{U} \rightarrow \Omega \cup B_{i}$ and $\pi^{\prime}$ : $\mathcal{U}^{\prime} \rightarrow \Omega^{\prime} \cup B_{i}^{\prime}$ be unbranched analytic covering maps, with deck transformation groups $\Gamma_{i}$ and $\Gamma_{i}^{\prime}$ respectively. Since $B_{i}$ and $B_{i}^{\prime}$ are contractible subsets of $\Omega \cup B_{i}$ and $\Omega^{\prime} \cup B_{i}^{\prime}$ respectively, the homeomorphism $f$ induces a homotopy equivalence $\Omega \cup B_{i} \rightarrow \Omega^{\prime} \cup B_{i}^{\prime}$, which in turn induces an isomorphism $\rho_{i}: \Gamma_{i} \rightarrow \Gamma_{i}^{\prime}$. Then $f$ lifts to a conformal homeomorphism

$$
\hat{f}_{i}: \mathcal{U} \backslash \pi^{-1}\left(B_{i}\right) \rightarrow \mathcal{U}^{\prime} \backslash \pi^{\prime-1}\left(B_{i}^{\prime}\right)
$$


Note that $\hat{f}_{i}$ is $\left(\Gamma_{i}, \Gamma_{i}^{\prime}\right)$-equivariant: for any element $\gamma \in \Gamma_{i}$ we have

$$
\rho_{i}(\gamma) \circ \hat{f}_{i}=\hat{f}_{i} \circ \gamma
$$

The connected components of $\pi^{-1}\left(B_{i}\right)$ and of $\pi^{\prime-1}\left(B_{i}^{\prime}\right)$ are disjoint closed discs because $\Omega$ and $\Omega^{\prime}$ are intrinsic circle domains. Therefore $\hat{f}_{i}$ is a conformal homeomorphism between circle domains. Hence by the uniqueness part of Theorem $B$ it is the restriction of a Möbius map $M_{i}$. We find that $M_{i}$ takes $\mathcal{U}$ onto $\mathcal{U}^{\prime}$ since each of the two circle domains has only one non-isolated boundary component. In particular, $\mathcal{U}=\mathcal{U}^{\prime}$. Moreover, the map $M_{i}: \mathcal{U} \rightarrow \mathcal{U}^{\prime}$ is also $\left(\Gamma_{i}, \Gamma_{i}^{\prime}\right)$-equivariant, since $\rho_{i}(\gamma) \circ M_{i} \circ \gamma^{-1}: \mathcal{U} \rightarrow \mathcal{U}^{\prime}$ is a Möbius map extending $\hat{f}_{i}$ and is therefore equal to $M_{i}$. It follows that $M_{i}$ descends to a conformal homeomorphism $\tilde{f}_{i}: \Omega \cup B_{i} \rightarrow \Omega^{\prime} \cup B_{i}^{\prime}$ that extends $f$.

Gluing the extensions $\tilde{f}_{i}$ together for $i=1, \ldots, n$, we obtain the desired conformal homeomorphism $\tilde{f}: S \rightarrow S^{\prime}$ extending $f$. This completes the proof of uniqueness.

\section{EXISTENCE}

The first step is to apply Theorem $\mathrm{B}$ to map $\Omega$ via a conformal homeomorphism onto a circle domain $\Omega^{*}$ in some compact Riemann surface $R$.

Our goal is to construct a quasiconformal homeomorphism $f$ of $R$ onto another Riemann surface $S$ so that $f$ is conformal on $\Omega^{*}$ and the image $f\left(\Omega^{*}\right)$ is an intrinsic circle domain in $S$. In fact, we will construct a Beltrami coefficient $\mu$ on $R$ with $\|\mu\|_{\infty}<1$; then by the measurable Riemann mapping theorem we will obtain a Riemann surface $S$ and a quasiconformal homeomorphism $f: R \rightarrow S$ such that $\mu(z) f_{z}=f_{\bar{z}}$ a.e. on $R$. By construction, $\mu$ will be identically zero on $\Omega^{*}$, so that $f$ is conformal there.

Consider any connected component $B$ of $R \backslash \Omega^{*}$ that is not a single point. Our aim is to construct $\mu$ on $R$ so that $f(B)$ will be a closed geometric disc with respect to the Riemann surface $f\left(\Omega^{*} \cup B\right)$. Since this is a condition on the conformal structure of $f\left(\Omega^{*} \cup B\right)$, it depends only on the restriction of $\mu$ to $\Omega^{*} \cup B$, which by construction will be non-zero only on $B$. This is a key point, for it means that we can correct the conformal structure on each complementary component separately and the corrections will not interfere with each other. This is in contrast to the proof of Koebe's theorem by iterated Riemann mapping, where each complementary component has to be corrected infinitely many times and the required mapping is obtained in the limit. By hypothesis $\Omega^{*}$ has only finitely many complementary components in $R$, so to ensure that $\|\mu\|_{\infty}<1$ it will suffice that $\|\mu\|_{B}<1$ for each complementary component $B$.

Let $\pi: \mathcal{U} \rightarrow \Omega^{*} \cup B$ be an unbranched analytic covering map, where $\mathcal{U}$ is one of $\mathbb{D}, \mathbb{C}$, or $\widehat{\mathbb{C}}$. We rule out the case $\mathcal{U}=\widehat{\mathbb{C}}$, since in this case $\Omega^{*}$ is an open disc in $\widehat{\mathbb{C}}$, so it is already an intrinsic circle domain. Let $\Gamma$ be the deck transformation group of $\pi$, i.e., the (infinite) group of conformal automorphisms $\gamma: \mathcal{U} \rightarrow \mathcal{U}$ such that $\pi \circ \gamma=\pi$. The preimage $\pi^{-1}(B)$ has infinitely many connected components, each of which is a topological closed disc, bounded by an analytic Jordan curve.

Apply Theorem A to the domain $\pi^{-1}\left(\Omega^{*}\right)=\mathcal{U} \backslash \pi^{-1}(B)$. This provides a conformal mapping $h: \pi^{-1}\left(\Omega^{*}\right) \rightarrow V \subset \widehat{\mathbb{C}}$, where $V$ is a circle domain. Each component of $\pi^{-1}(B)$ corresponds under $h$ to a component of $\widehat{\mathbb{C}} \backslash V$ that is a closed disc, not a singleton, since it is isolated and cannot be separated from the other 


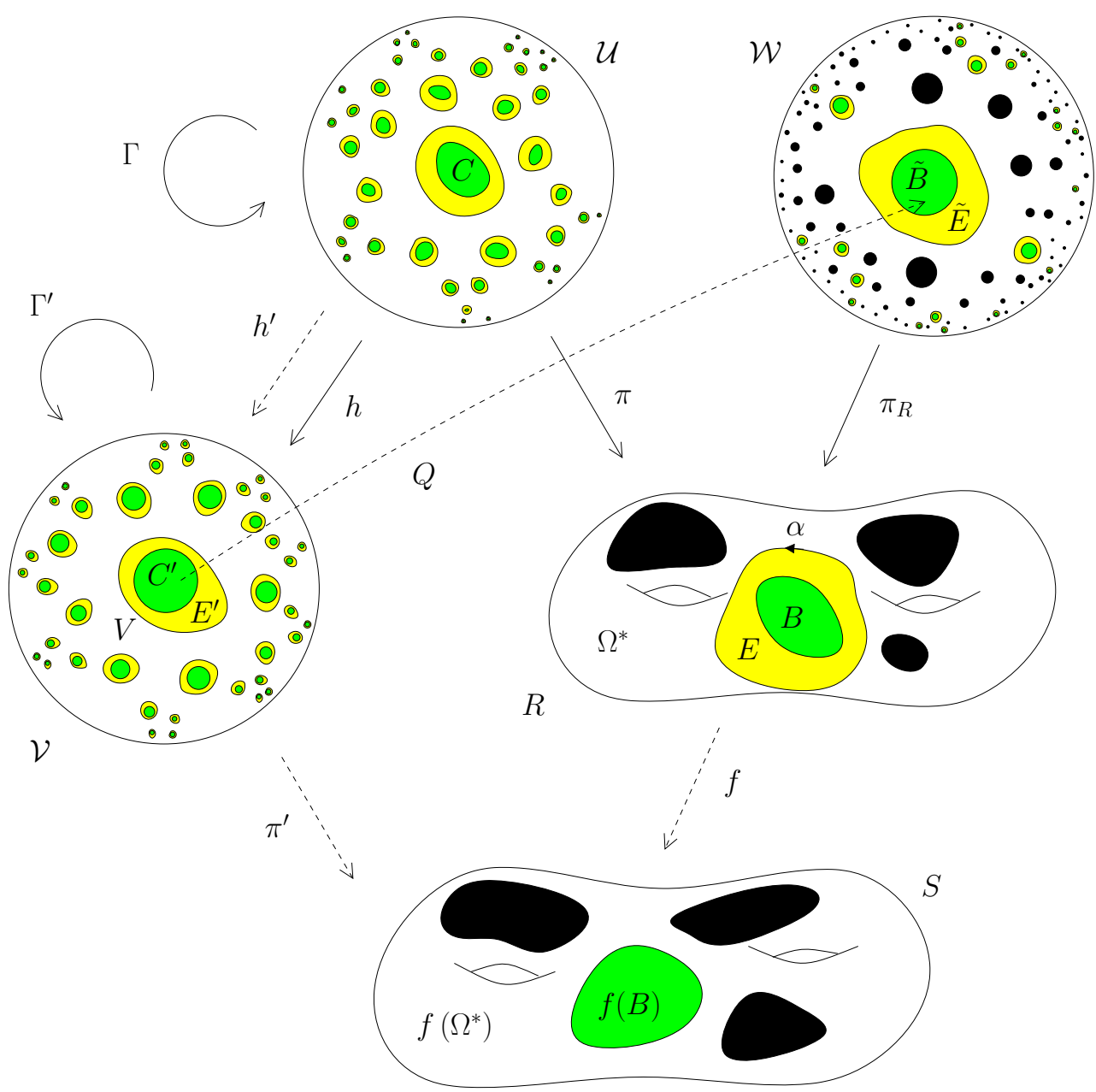

Figure 1. Domains, maps and group actions in the existence proof, illustrating the case where $R$ is hyperbolic.

components by a ring domain of arbitrarily large modulus contained in $\pi^{-1}\left(\Omega^{*}\right)$. The remaining end of $\pi^{-1}\left(\Omega^{*}\right)$ may correspond to either a point or a disc in the complement of $V$; this component is distinguished because it is not isolated. Let $\mathcal{V}$ be the union of $V$ and all of its isolated complementary components.

For any non-trivial element $\gamma \in \Gamma$, the map $h \circ \gamma$ is also a conformal mapping of $\pi^{-1}\left(\Omega^{*}\right)$ onto the circle domain $V$, so by the uniqueness part of Theorem $\mathrm{A}$ we have $h \circ \gamma=\gamma^{\prime} \circ h$ for a unique Möbius map $\gamma^{\prime}$, which restricts to an automorphism of $V$ with no fixed points since $\gamma$ has no fixed points in $\mathcal{U}$. The map that sends $\gamma$ to $\gamma^{\prime}$ is therefore an injective homomorphism $\Gamma \rightarrow \Gamma^{\prime}$, where $\Gamma^{\prime}$ is a subgroup of $\operatorname{Aut}(\mathcal{V})$. Since $\Gamma^{\prime}$ acts simply transitively on the components of $h\left(\pi^{-1}(B)\right)$ and has no fixed points in $V$, it acts freely on $\mathcal{V}$. Since $\operatorname{Aut}(V)$ acts properly discontinuously on $V$, and each compact subset of $\mathcal{V}$ intersects only finitely many components of $h\left(\pi^{-1}(B)\right)$, we also conclude that $\Gamma^{\prime}$ acts properly discontinuously on $\mathcal{V}$. 
Let $C$ be a connected component of $\mathcal{U} \backslash \pi^{-1}\left(\Omega^{*}\right)$, and let $C^{\prime}$ be the corresponding component of $\mathcal{V} \backslash V$, so that $C^{\prime}$ is bounded by a circle $\partial C^{\prime}$. Let $\pi_{R}: \mathcal{W} \rightarrow R$ be an analytic universal covering of $R$, so that $\pi_{R}^{-1}\left(\Omega^{*}\right)$ is a circle domain. Let $\tilde{B}$ be a connected component of $\pi_{R}^{-1}(B)$, bounded by a circle $\partial \tilde{B}$.

We will examine the behavior of the maps $h, \pi$ and $\pi_{R}$ in the neighborhood of the curves $\partial C$ and $\partial \tilde{B}$. For the present proof the aim is merely to show that $h$ may be extended continuously to a quasiconformal homeomorphism $h^{\prime}: \mathcal{U} \rightarrow \mathcal{V}$ that is equivariant with respect to $\Gamma$ and $\Gamma^{\prime}$. In fact, we will work slightly harder, using Lemma 3 to show that the quasiconformal dilatation of this extension can be made to depend only on a conformal invariant of $\Omega$. This will be useful in the next section.

Let $\alpha$ be the simple closed geodesic in the hyperbolic metric of $\Omega^{*}$ that separates $B$ from all the other components of $R \backslash \Omega^{*}$. Let $E$ be the ring domain bounded between $\alpha$ and $B$. It has $\operatorname{modulus} \bmod (E)=\pi^{2} / 2 \ell(\alpha)$, where $\ell(\alpha)$ is the length of $\alpha$ in the hyperbolic metric of $\Omega^{*}$; note that this length is a conformal invariant of $\Omega$.

The restriction of $\pi_{R}$ to this circle domain is an unbranched analytic covering of $\Omega^{*}$ and is therefore an isometry from the hyperbolic metric of $\pi_{R}^{-1}\left(\Omega^{*}\right)$ to the hyperbolic metric of $\Omega^{*}$. Thus the connected components of $\pi_{R}^{-1}(\alpha)$ are simple closed geodesics in the hyperbolic metric of $\pi_{R}^{-1}\left(\Omega^{*}\right)$ and are therefore disjoint, each separating a connected component of $\pi_{R}^{-1}(B)$ from all of the other components of $\mathcal{W} \backslash \pi_{R}^{-1}\left(\Omega^{*}\right)$. It follows that $\pi_{R}$ maps each connected component of $\pi_{R}^{-1}(E)$ bijectively onto $E$. One of these is a ring domain $\tilde{E}$ surrounding $\tilde{B}$.

Likewise, the restriction of $\pi \circ h^{-1}$ to $V$ is an unbranched analytic covering of $\Omega^{*}$, so $\pi \circ h^{-1}$ maps each connected component of $h \circ \pi^{-1}(E)$ bijectively onto $E$. One of these connected components is a ring domain $E^{\prime}$ surrounding $C^{\prime}$.

Consider the branch of $\pi_{R}^{-1} \circ \pi \circ h^{-1}$ that maps $E^{\prime}$ to $\tilde{E}$. It is a homeomorphism of ring domains taking the inner boundary circle $\partial C^{\prime}$ onto the inner boundary circle $\partial \tilde{B}$. Lemma 3 gives an extension to a quasiconformal homeomorphism $Q$ from $E^{\prime} \cup C^{\prime}$ to $\tilde{E} \cup \tilde{B}$.

Now $Q^{-1} \circ \pi_{R}^{-1}$ gives us a quasiconformal homeomorphism $g: B \rightarrow C^{\prime}$ that continuously extends the boundary correspondence induced by of $h \circ \pi^{-1}$. We define $\left.\mu\right|_{B}$ to be the Beltrami coefficient $g_{\bar{z}} / g_{z}$.

When we solve the Beltrami equation to obtain $f: R \rightarrow S$, the covering map $\pi^{\prime}: \mathcal{V} \rightarrow\left(S \backslash f\left(\Omega^{*}\right)\right) \cup f(B)$ such that $f \circ \pi=\pi^{\prime} \circ h$ will be an unbranched analytic covering, mapping the disc $C^{\prime}$ onto $f(B)$, as required.

\section{The CASE OF COUntably Infinite CONNECTIVITY}

It is natural to ask whether the notion of intrinsic circle domains can be extended to domains of countably infinite connectivity.

5.1. All ends isolated. First, we deal with the case of a domain $\Omega$ such that every end of $R$ is isolated, i.e., has a neighborhood that meets no other end. If $\Omega$ is embedded in a compact ambient Riemann surface, then this implies that $\Omega$ has only finitely many ends and finite genus, and we already understand this case.

For a more interesting example, let $R$ be a $\mathbb{Z}$-cover of a compact genus 2 Riemann surface, so that $R$ has infinite genus and two ends. Then let $\Omega$ be a domain obtained by removing countably many disjoint closed topological discs with no accumulation 
point from $R$. We could hope to modify the structure of $R$ on a neighborhood of each disc in order to make the complementary components be intrinsic discs. However, there is no way to fix the two ends of $R$ so that they are represented by intrinsic discs, for the resulting ambient Riemann surface would be compact and therefore have only finite genus.

Suppose that $\Omega$ is infinitely connected or has infinite genus. We call an end of $\Omega$ fixable if it has a neighborhood that is a ring domain.

Lemma 4. For any Riemann surface $\Omega$, there is a conformal embedding of $\Omega$ into another Riemann surface $R$ such that every connected component of $R \backslash \Omega$ is an intrinsic disc and no end of $R$ is fixable.

Proof. We can find disjoint open neighborhoods of all the fixable ends, for example, by cutting along closed geodesics or horocycles around each end.

In the existence proof for Theorem 2 we only used a local surgery to modify the ambient Riemann surface in a neighborhood of each complementary component. The initial step of passing to a circle domain was technically convenient (and will be needed later), but was not really necessary. All we really needed to know was that for each individual end $E$, we can embed $\Omega$ in a Riemann surface $R_{E}$ such that $R_{E} \backslash \Omega$ is a geometric disc in $R_{E}$. This is true for any fixable end $E$, since it has a neighborhood that is a ring domain and therefore it has a neighborhood $U_{E}$ conformally equivalent to a round annulus $A=\{z \in \mathbb{C}|a<| z \mid<1\}$, for some $0 \leq a<1$. Then we can glue the open unit disc to $\Omega$, identifying $A$ with $U_{E}$, to obtain a Riemann surface $R_{E}^{\prime}$ in which $\Omega$ is embedded so that the end $E$ corresponds to a connected component $K$ of $R_{E}^{\prime} \backslash \Omega$. Then we can apply Theorem A to the preimage of $\Omega$ in the universal cover of $R_{E}^{\prime}$, to find out how to modify the conformal structure on $K$ to obtain an embedding of $\Omega$ in a new Riemann surface $R_{E}$ so that the end $E$ corresponds to a connected component of $R_{E} \backslash \Omega$ that is an intrinsic disc.

To construct $R$ we glue together all the Riemann surfaces $R_{E}$ corresponding to fixable ends $E$ by identifying the embedded copies of $\Omega$. The resulting $R$ is locally a Riemann surface, is connected, and is second countable because $\Omega$ can only have countably many fixable ends.

5.2. Non-isolated ends. We now return to the case of a subdomain $\Omega$ of a compact Riemann surface, but assume that $\Omega$ is countably infinitely connected. It must now be the case that some complementary components are not isolated. There is a potential topological obstruction associated with complementary components that are not isolated: they cannot be represented by intrinsic discs of finite radius. We might attempt to salvage something by allowing complementary components to be horodiscs with respect to the hyperbolic metric, but this would not help in the case of a circle domain $\Omega \subset \widehat{\mathbb{C}}$ in which some circular complementary component $B$ has precisely two points on its boundary that are accumulation points of other complementary circles.

Therefore in the countably infinitely connected case, we define an intrinsic circle domain $\Omega$ to be a subdomain of a compact Riemann surface $R$ such that any complementary component that is not isolated is a singleton and every non-singleton complementary component $L$ is a closed geometric disc with respect to $\Omega \cup L$. This definition is intended to be analogous to the definition of a circle domain in the countably connected case. 
In order to obtain a positive theorem, we can place a simple conformal geometric constraint on the domain. We will say that a countably connected domain $\Omega$ in a compact Riemann surface $R$ is uniformly separated if there exists $\epsilon>0$ such that each connected component of $R \backslash \Omega$ either is a single point or is separated from all the remaining complementary components by a ring domain of modulus at least $\epsilon$ embedded in $R \backslash \Omega$.

Lemma 5. Suppose $\Omega$ is a countably-connected uniformly separated domain in a compact Riemann surface $R$. Let $\varphi$ be any conformal embedding of $\Omega$ into a compact Riemann surface $R^{\prime}$ of the same genus as $R$. Then the non-singleton complementary components of $R^{\prime}$ correspond to the non-singleton complementary components of $R$, so $\varphi(\Omega)$ is uniformly separated in $R^{\prime}$.

To explain the significance of this, we first note that a complementary component $B$ is isolated if and only if $\Omega$ contains a ring domain with $B$ as one of its complementary components; this is a topological condition on $\Omega$, so the corresponding complementary component in $\varphi(\Omega)$ is also not isolated. If $\Omega$ is uniformly separated, then the connected components of $R \backslash \Omega$ that are not singletons must be isolated. However, the remaining complementary components need not be punctures; some or all of them could be accumulation points of sequences of other complementary components. For a general open Riemann surface $\Omega$ of countable connectivity and finite genus, it is possible for an end of $\Omega$ to be represented in one conformal embedding by a complementary component that is a non-isolated singleton, yet in some other conformal embedding to be represented by a non-singleton.

We can construct an example of this cavitation behaviou 1$]$ as follows. Consider a subdomain $D$ of $\mathbb{C} \backslash \overline{\mathbb{D}}$ obtained by removing countably many complex conjugate pairs of circular arcs, where one arc of the $n^{\text {th }}$ pair is

$$
A_{n}=\left\{\left(1+2^{-n}\right) e^{i t}: \theta_{n}<t<\pi-\theta_{n}\right\}, \quad \theta_{n} \searrow 0 .
$$

The domain

$$
D_{n}=\mathbb{C} \backslash\left(\mathbb{D} \cup \bigcup_{j=1}^{n}\left(A_{j} \cup \overline{A_{j}}\right)\right)
$$

is conformally equivalent via a conformal map that fixes $z=2$ to a slit domain $S_{n}$, which may be obtained from $\mathbb{C} \backslash\{0\}$ by removing finitely many pairs of intervals $I_{n}$, $\overline{I_{n}}$ contained in the imaginary axis, arranged symmetrically about 0 , together with an interval $\left(-\delta_{n} i, \delta_{n} i\right)$. See Figure 2 for a schematic illustration. One can show that if the angles $\theta_{n}$ are chosen to decrease to 0 sufficiently fast, then the domains $S_{n}$ converge in the Carathéodory topology to a limit domain $S$ in which 0 is a non-isolated singleton complementary component, but every other complementary component is an isolated interval of the imaginary axis. Then $D$ is conformally equivalent to $S$ and we have an example of cavitation: the singleton complementary component $\{0\}$ of $S$ corresponds to the complementary component $\overline{\mathbb{D}}$ of $D$.

The idea is that by making $\theta_{n}$ small we can ensure that the extremal length of the family of curves in $D$ joining $A_{n}$ to $\overline{A_{n}}$ is as small as we like. This means we can make the gap between the intervals $I_{n}$ and $\overline{I_{n}}$ as short as we like in comparison to the length of $I_{n}$. In fact, to ensure that the end represented in $D$ by the unit circle is represented in $S$ by a singleton, it suffices to take $\theta_{n}=2^{-n}$.

\footnotetext{
${ }^{1}$ The term cavitation refers in fluid dynamics to the sudden formation of a bubble as a dissolved gas comes out of solution around a nucleation site.
} 

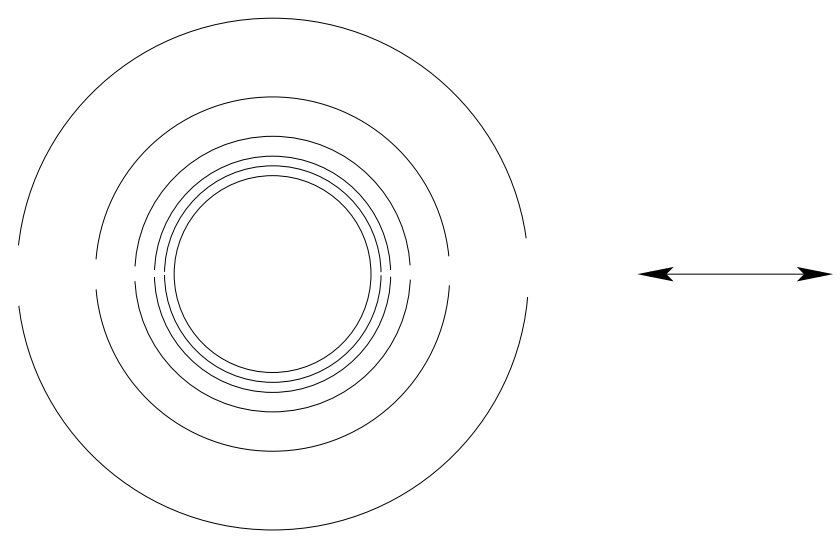

Figure 2. Two conformally equivalent countably connected domains.

We will now prove Lemma 5, which says that uniform separation prevents cavitation.

Proof. Suppose each non-singleton connected component $B_{i}$ of $R \backslash \Omega$ is separated from the remaining complementary components by a ring domain $A_{i} \subseteq \Omega$ of modulus $\epsilon$. Then the core curve $\gamma_{i}$ of the ring domain $A_{i}$ has length $2 \pi^{2} / \epsilon$ with respect to the hyperbolic metric of $A_{i}$. By the Schwarz-Pick lemma, the length of $\gamma_{i}$ with respect to the hyperbolic metric of $\Omega$ is also at most $2 \pi^{2} / \epsilon$. Consider the simple closed geodesic $g_{i}$ (with respect to the hyperbolic metric of $\Omega$ ) that lies in the free homotopy class of $\gamma_{i}$. Since $g_{i}$ is a length minimizer in its free homotopy class, it is no longer than $\gamma_{i}$ in the hyperbolic metric of $\Omega$. It follows that the ring domain $E_{i}$ bounded between $g_{i}$ and $B_{i}$ has modulus at least $\epsilon / 4$. Our reason for passing from the ring domains $A_{i}$ to the ring domains $E_{i}$ is that the closures of the $E_{i}$ in $R$ are pairwise disjoint, which was not necessarily true of the $A_{i}$, and moreover, the $E_{i}$ are intrinsically-defined subdomains of $\Omega$.

We now cut $\Omega$ along each $g_{i}$ to leave a domain $U=\Omega \backslash \bigcup \overline{E_{i}}$. We claim that for any conformal embedding $\varphi$ of $\Omega$ into a compact Riemann surface $R^{\prime}$ of the same genus as $R$, there exists a quasiconformal homeomorphism $Q: R \rightarrow R^{\prime}$ such that $\left.\varphi\right|_{U}=\left.Q\right|_{U}$. To prove this, consider a component $D_{i}=B_{i} \cup E_{i}$ of $R \backslash U$. It corresponds to a component $D_{i}^{\prime}=B_{i}^{\prime} \cup \varphi\left(E_{i}\right)$ of $R^{\prime} \backslash \varphi(U)$, where $B_{i}^{\prime}$ is a component of $R^{\prime} \backslash \varphi(\Omega)$. We claim that there is a $K$-quasiconformal homeomorphism $D_{i} \rightarrow D_{i}^{\prime}$ whose restriction to the curve $g_{i}$ agrees with $\varphi$. Here $K$ depends only on $\epsilon$. To see this, let $\psi: D_{i} \rightarrow \mathbb{D}$ and $\chi: D_{i}^{\prime} \rightarrow \mathbb{D}$ be Riemann mappings, and note that $\partial D_{i}$ and $\partial D_{i}^{\prime}$ are analytic Jordan curves. Apply Lemma 3 to the conformal homeomorphism

$$
\chi \circ \varphi \circ \psi^{-1}: \psi\left(E_{i}\right) \rightarrow \chi\left(E_{i}^{\prime}\right)
$$


and apply a Schwarz reflection to obtain a $K$-quasiconformal homeomorphism $h$ : $\mathbb{D} \rightarrow \mathbb{D}$ whose boundary correspondence agrees with that of $\chi \circ \varphi \circ \psi^{-1}$, where $K$ depends only on $\epsilon$. Now

$$
\chi^{-1} \circ h \circ \psi: D_{i} \rightarrow D_{i}^{\prime}
$$

is a $K$-quasiconformal homeomorphism agreeing with $\varphi$ on the boundary, as required.

Gluing together $\varphi$ and the $K$-quasiconformal extensions to the components $D_{i}$, we obtain a $K$-quasiconformal homeomorphism

$$
H: \Omega \cup \bigcup B_{i} \rightarrow \varphi(\Omega) \cup \bigcup B_{i}^{\prime} .
$$

Since $\Omega$ is countably connected, the complement $X=R \backslash\left(\Omega \cup \cup B_{i}\right)$ is a countable union of singletons, and therefore is a removable set for the solution of the Beltrami equation. Thus $H$ extends to a homeomorphism $R \rightarrow R^{\prime}$, and hence the complementary components of $R^{\prime}$ that are not among the $B_{i}$ are singletons.

Theorem 6. Suppose $\Omega$ is a domain in a compact Riemann surface $R$ such that $\Omega$ has countably infinite connectivity, has the same genus as $R$, and is uniformly separated. Then there is a conformal embedding $\varphi$ of $\Omega$ in a compact Riemann surface $S$ so that $\varphi(\Omega)$ is an intrinsic circle domain in $S$. The pair $(S, \varphi)$ is unique up to conformal isomorphism.

Proof. The existence proof is similar to that of Theorem 2 Lemma 5 shows that when we apply Theorem $\mathrm{B}$, the resulting countably connected circle domain $\Omega^{*} \subset R$ satisfies the hypotheses of Theorem [6, in addition to having the property that its non-singleton complementary components are geometric discs. Then we note that by Lemma 3 the quasiconformal distortion of the extension $C \rightarrow B$ can be bounded in terms of $\epsilon$. Then the resulting Beltrami coefficient $\mu$ has $\|\mu\|_{\infty}<1$, so we may still apply the measurable Riemann mapping theorem to obtain the desired conformal structure on $S$. Then Lemma 5 ensures that the non-isolated complementary components of $f\left(\Omega^{*}\right)$ are singletons, so that $f\left(\Omega^{*}\right)$ is an intrinsic circle domain.

The uniqueness proof goes through as before, but with one additional step. Because of the separation hypothesis there is no difficulty in extending a conformal homeomorphism $f: \Omega \rightarrow \Omega^{\prime}$ between two countably-connected intrinsic circle domains across each non-singleton complementary component. We thus obtain a conformal homeomorphism between domains whose complements consist of countably many points, which in turn extends to a conformal homeomorphism $\tilde{f}: S \rightarrow S^{\prime}$ between the ambient Riemann surfaces.

It is likely that the condition of uniform separation can be weakened by making use of results on existence and uniqueness of solutions to the Beltrami equation in the case where the Beltrami coefficient has norm 1 but the measure of the set on which the distortion is large is controlled.

\section{Mixtures of intrinsic AND EXtrinsic CirCles}

Now we present a generalization of Theorems 2 and 6 in which the conditions on the complementary components of $\Omega$ in $S$ are mixed. That is, some components are punctures or geometric discs in the natural geometry of the ambient compact Riemann surface $S$, while each of the others is an intrinsic disc, i.e., a geometric disc with respect to its own union with $\Omega$. 
Theorem 7. Let $\Omega$ be an open Riemann surface with finite genus and at most countably many ends. Let $K_{i}, i \in I$ be some of the ends of $\Omega$, none of them punctures, where the index set I may be either finite or countably infinite. Suppose that for some $\epsilon>0$ and for each $i \in I$ there is a ring domain $A_{i}$ contained in $\Omega$, with modulus at least $\epsilon$, that separates the end $K_{i}$ from the other ends of $\Omega$. Then $\Omega$ is conformally equivalent to a domain $\varphi(\Omega)$ in a compact Riemann surface $S$, with complementary components $L_{i}$ corresponding to $K_{i}$, such that $\varphi(\Omega) \cup \bigcup_{i \in I} L_{i}$ is a circle domain in $S$ and for each $i \in I, L_{i}$ is either a singleton or a closed geometric disc with respect to the domain $\varphi(\Omega) \cup L_{i}$. Such a pair $(S, \varphi)$ is unique up to conformal isomorphism.

Proof. We begin by replacing $\Omega$ by a conformally equivalent circle domain $\Omega^{*}$ in a compact Riemann surface $R$, as in Theorem B. Apply the construction of section 4 to each of the components $K_{i}, i \in I$, to obtain a Beltrami differential $\mu(z) \frac{d z}{d \bar{z}}$ supported on $\bigcup K_{i}$. In the case where $I$ is infinite, the separation condition guarantees that $\|\mu\|_{\infty}<1$, as it did for Theorem [6. Solving the Beltrami equation $\mu(z) f_{z}=f_{\bar{z}}$ on $R$ gives a new compact Riemann surface $S^{\prime}$ with a homeomorphism $f: R \rightarrow R^{\prime}$, conformal away from the preimages of the $K_{i}$, such that $f\left(K_{i}\right)$ is a closed hyperbolic disc with respect to the hyperbolic metric of $f(U) \cup f\left(K_{i}\right)$. The domain $f\left(U \cup \cup K_{i}\right)$ is countably connected so by Theorem $\mathrm{B}$ there is a conformal homeomorphism $g: f\left(U \cup \cup K_{i}\right) \rightarrow \Omega^{*}$, where $\Omega^{*}$ is a circle domain in a compact Riemann surface $S$. The image $\Omega=g(f(U))$ is the required domain, and $L_{i}=g\left(f\left(K_{i}\right)\right)$. Indeed, $g$ is conformal on $U \cup f\left(K_{i}\right)$, so it is an isometry from the hyperbolic metric of $U \cup f\left(K_{i}\right)$ to the hyperbolic metric of $\Omega \cup L_{i}$.

To prove uniqueness, suppose that $f: \Omega \rightarrow \Omega^{\prime}$ is a conformal homeomorphism between two domains $\Omega \subset S$ and $\Omega^{\prime} \subset S^{\prime}$, each satisfying the conditions of the theorem. Let the complementary components of $\Omega^{\prime}$ be $L_{i}^{\prime}, i \in I$. Apply the argument of $\$ 3$ to extend $f$ to a conformal homeomorphism of countably connected circle domains $f: \Omega \cup \bigcup L_{i} \rightarrow \Omega^{\prime} \cup \bigcup L_{i}^{\prime}$. This must be the restriction of a conformal map from $S$ to $S^{\prime}$ by the final part of He and Schramm's Theorem B.

\section{Numerical APPROXimation USING CIRClE PACKINGS}

The rest of this paper concerns the practical numerical approximation of finitelyconnected intrinsic circle domains in the sphere. The idea is roughly to follow the steps of the existence proof, performing each step numerically.

First, we need a way to approximate circle domains having Fuchsian symmetry. This already presents us with a choice of methods, as there are several numerical methods for computing circle domains. Finitely connected circle domains can be approximated using iterated Riemann mapping, the individual steps of which can be carried out using a number of different methods for computational conformal mapping. However, we must deal with an infinitely connected circle domain, so it seems at first sight that we must make decisions about which complementary components to ignore at any particular stage of the approximation. We will see that this truncation can in fact be avoided.

Second, if we follow the existence proof directly, it seems that we need to compute a quasiconformal extension numerically, find its dilatation and then solve a Beltrami equation on a larger domain. In practice, these steps can be combined into a single conformal welding step. 
A final practical difficulty may arise in converting the output of each step into the input for the next step, especially when the steps require different types of grid.

Fortunately, circle packing is a numerical approximation method that offers solutions to all of these challenges. Although circle packing is not often used for high-precision conformal mapping problems on account of its relatively slow convergence, it is particularly well suited to the approximation of circle domains, especially those with Fuchsian symmetry. It is also a practical tool for numerical conformal welding. We were able to carry out all of the steps of an approximation procedure for computing intrinsic circle domains using the CirclePack software [1] written by Ken Stephenson et al. For an introduction to circle packing and its use as a computational tool, we refer the reader to Stephenson's monograph [10].

7.1. Overview of circle packing. Let $T$ be a finite graph embedded in the plane. A circle packing of $T$ in the plane is a collection $\mathcal{P}$ of circular discs $C_{v}$ with disjoint interiors, one corresponding to each vertex $v$ of $T$, such that whenever vertices $v$ and $w$ are adjacent in $T$ the $\operatorname{discs} C_{v}$ and $C_{w}$ are tangent. We call $T$ the nerve of $\mathcal{P}$. We may also circle pack in the sphere, using discs in the spherical metric, or in the hyperbolic plane, using hyperbolic circles and possibly also horocircles on the boundary. The carrier of $\mathcal{P}$ is the geometric complex formed by connecting the centers of neighboring circles by geodesic segments. According to the KoebeAndreev-Thurston theorem, when $T$ is any triangulation of the sphere, there exists a circle packing of $T$, and it is unique up to Möbius maps and reflection. In this case, the carrier of $\mathcal{P}$ is the entire sphere. Since we want to use circle packings to approximate conformal structures, we remove the reflection ambiguity by imposing a fixed orientation.

There is a beautiful algorithm due to Bill Thurston for computing a circle packing from a given nerve. It works by removing one vertex and packing the remaining triangulation into the unit disc. This is achieved by computing the hyperbolic packing label for the packing; this is the function which assigns a hyperbolic radius to each vertex of the triangulation in such a way that the boundary circles are given infinite radius so that they correspond to horocircles, are internally tangent to the unit circle, and all interior circles have finite hyperbolic radius. There is a unique packing label that results in an angle sum of $2 \pi$ at each interior vertex. Thurston's algorithm approximates the correct label as the limit of a pointwise increasing sequence of labels. Once the correct packing label is known, the circles of the given radii can be laid out in the hyperbolic plane iteratively so that each satisfies the appropriate tangency conditions.

7.2. Outline of the approximation algorithm. Here we outline a numerical method for approximating intrinsic circle domains. Let $\Omega$ be a given finitely connected domain in the Riemann sphere. Begin by taking a circle packing $\mathcal{P}$ such that the interiors of all the circles of $\mathcal{P}$ are contained in $\Omega$ and the complementary components of $\Omega$ are separated by the carrier of $\mathcal{P}$. All subsequent calculations depend only on the nerve of $\mathcal{P}$, so it is in this step that we have captured a discrete approximation to the conformal equivalence class of $\Omega$. The quality of the final approximation of the intrinsic circle domain $\Omega^{*}$ conformally equivalent to $\Omega$ will depend on the mesh of $\mathcal{P}$ (the size of its largest circle) and the maximum distance of $\partial \Omega$ from the carrier of $\mathcal{P}$. 
We perform a sequence of circle packing computations in order to construct a triangulation of the sphere together with an embedding of the nerve of $\mathcal{P}$ as a subcomplex. When we compute the circle packing $\mathcal{Q}$ of the spherical triangulation, the carrier of the embedded sub-packing gives an approximation to the intrinsic circle domain $\Omega$ conformally equivalent to $U$. We can interpolate the mapping of circle centers of $\mathcal{P}$ to the corresponding circle centers in $\mathcal{Q}$ to give a polyhedral embedding of the carrier of $\mathcal{P}$ into the carrier of $\mathcal{Q}$. Since we are mapping from the plane to the sphere, we cannot map in a piecewise affine fashion, so instead we map in a piecewise affine fashion to the polyhedron in $\mathbb{R}^{3}$ whose vertices are the vertices of $\mathcal{Q}$ in $S^{2}$, and then project radially outwards to $S^{2}$. This map gives a homeomorphism which approximates the conformal map from $U$ to $\Omega$ and is locally quasiconformal.

We will explain informally why each step of the computation provides an arbitrarily good approximation to a conformal map occurring at the corresponding step in the constructive proof of Theorem 1. Note that the computation consists of finitely many circle packing steps. In each step we are approximating the solution of a problem whose solution depends continuously on its data. This should suffice to prove local uniform convergence of the approximations to the conformal map from $\Omega$ to $\Omega^{*}$, but we will not attempt to estimate the rate of convergence.

7.3. Approximating circle domains via circle packing. Suppose we are given a bounded finitely connected domain $\Omega$ in the complex plane. We find a sequence of circle packings $\mathcal{P}_{n}$ in $\Omega$ whose carriers exhaust $\Omega$. To construct $\mathcal{P}_{n}$ we cut out a portion of the regular hexagonal circle packing with circles all of radius $\epsilon=2^{-n}$. This means that we keep only those circles whose interiors are entirely contained in $\Omega$. Stephenson [10] describes this as using $\partial \Omega$ as a "cookie cutter". We have to take some care at the boundary: we retain only the largest connected component of the hexagonal packing that remains, discarding any peripheral islands and iteratively removing vertices of degree one until we are left with a connected triangulation. When we take $n$ large enough, the boundary components of this triangulation will be in one-to-one correspondence with the complementary components of $\Omega$.

Next, to each cycle of boundary vertices of $\mathcal{P}_{n}$ we adjoin a new "ideal" vertex, adjacent to all of the vertices in the cycle, and in this way we obtain a triangulation of the sphere. By the Koebe-Andreev-Thurston theorem there is a circle packing whose nerve is this triangulation, and it is unique up to Möbius transformations. Consider what happens to this spherical packing as we let $n$ tend to $\infty$. Using quasiconformal distortion estimates for packings of bounded vertex degree, one can show that after a suitable Möbius normalization, the circles corresponding to the added ideal vertices converge to limit circles of positive radius, while the maximum radius of the other circles tends to zero uniformly.

Let $f_{n}$ be the polyhedral map from the hexagonal cut-out packing $\mathcal{P}_{n}$ to the carrier of the spherical packing, normalized so that three chosen points in $\Omega$ are fixed by $f_{n}$. It turns out that as $n \rightarrow \infty, f_{n}$ converges locally uniformly on $\Omega$ to a conformal map from $\Omega$ to a circle domain. To prove this, we can use the hex packing lemma of Rodin and Sullivan [7. This says that the quasiconformal dilatation of $f_{n}$ converges locally uniformly to 0 on $\Omega$. The Rodin-Sullivan bound for the quasiconformal dilation on a given face of the triangulation depends only on the number of layers of hexagonal packing surrounding it, and tends to 0 as the number of layers tends to infinity. Let $\Omega^{\prime}$ be any subdomain of $\Omega$ bounded 
by Jordan curves in $\Omega$, such that $\Omega^{\prime}$ is homeomorphic to $\Omega$. The dilatation bound shows that the images of the maps $f_{n}$ restricted to $\Omega^{\prime}$ remain within a compact subset of the Teichmüller space of multiply connected domains homeomorphic to $\Omega$. Any subsequential limit of the sequence $f_{n}$ must be a conformal homeomorphism, and its image must be a circle domain. Given the normalization, there is a unique such homeomorphism. It follows that the sequence $f_{n}$ converges locally uniformly as $n \rightarrow \infty$.

We can augment the image packings by inverting across all the "ideal" circles, adding circles of degree 4 in the resulting four-sided interstices to maintain a triangulation. By considering moduli of ring domains in the resulting packing it is possible to show that the maximum radius of any non-ideal circle in the image packing also converges to 0 as $n \rightarrow \infty$.

We have now seen how to compute an arbitrarily good approximation to the circle domain $\Omega^{*}$ conformally equivalent to $\Omega$. We may assume that $\Omega^{*}$ is contained in the plane.

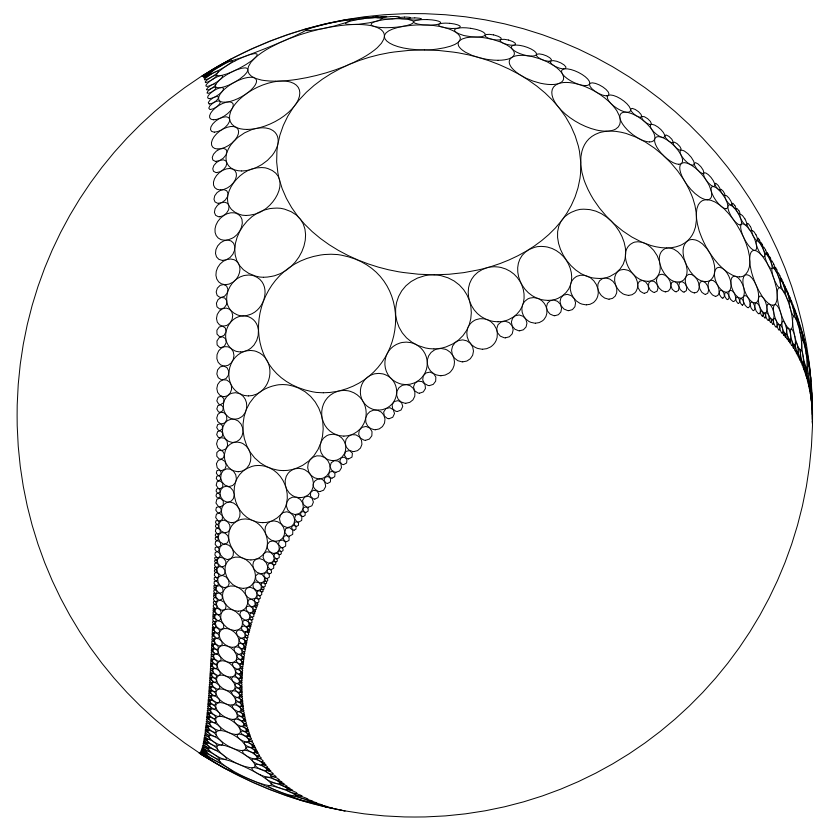

FiguRE 3. A circle packing of a circle domain in the Riemann sphere. Both the domain and the circle packing have sixfold symmetry.

7.4. Circle domains with Fuchsian symmetry. Suppose $B$ is one of the complementary components of $\Omega^{*}$. Let $\pi: \mathbb{D} \rightarrow \Omega^{*} \cup B$ be an analytic universal covering map. To compute an approximation to the circle domain conformally equivalent to $\mathbb{D} \backslash \pi^{-1}(B)$, we use the same trick again. We have a triangulation of $\Omega^{*}$, to which we add just one ideal vertex $v_{B}$ corresponding to the complementary component $B$. 
Now we apply Thurston's algorithm to compute the hyperbolic packing label for the resulting multiply connected triangulation. If we lay the circles out according to this packing label, we get a Fuchsian monodromy group. Call the resulting packing $\mathcal{P}_{B}$. The circle corresponding to $v_{B}$ and its translates under the monodromy group approximate the bounded components of the required circle domain. For the conformal welding step that follows we will only need to know the hyperbolic radii for the circles corresponding to the vertex $v_{B}$ and its neighbors. It is not necessary to perform the layout routine.

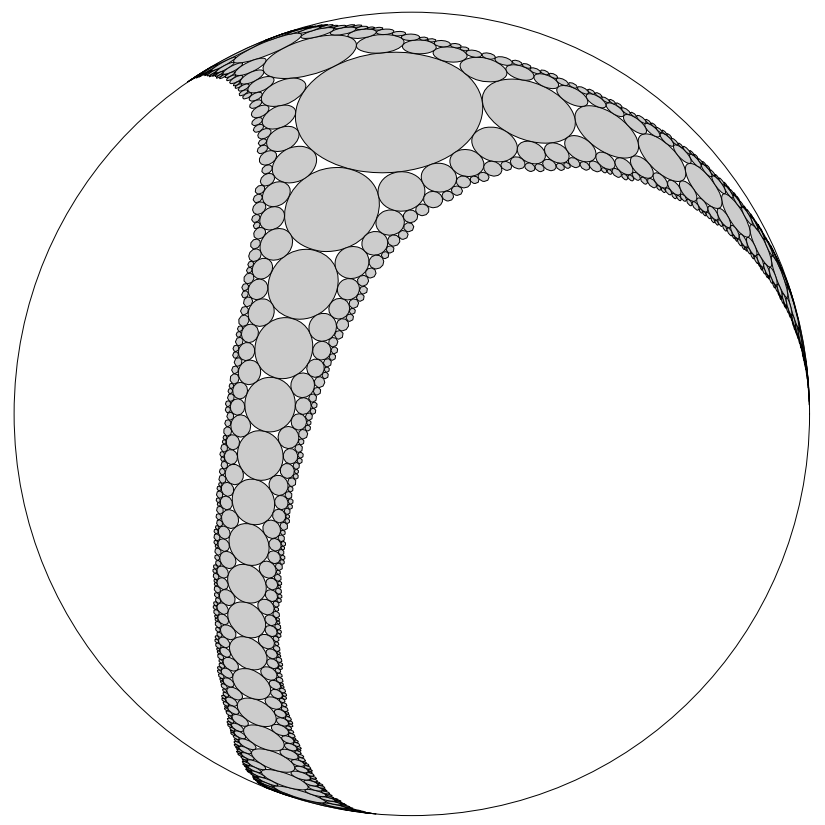

Figure 4. A circle packing approximation of the intrinsic circle domain conformally equivalent to the circle domain in Fig. 3, with the circle packing of the same complex shown.

7.5. Conformal welding via circle packing. Given a Jordan curve $\gamma$ in the Riemann sphere, we can compute the Riemann mappings of the unit disc onto the interior and the exterior of $\gamma$. The boundary values of these Riemann mappings give two different continuous maps of the unit circle onto $\gamma, \phi_{\text {int }}$ and $\phi_{\text {ext }}$. The shape of $\gamma$ is encapsulated in the boundary correspondence $\phi_{\text {ext }}^{-1} \circ \phi_{\text {int }}$, which is an orientation-reversing homeomorphism of the unit circle. Conformal welding is the process of recovering $\gamma$ (up to a Möbius map) from the boundary correspondence.

The CirclePack software includes a package for performing approximate conformal welding, following the procedure described by Williams [12. The idea is to paste two combinatorial closed discs together along their boundaries, according to a best possible combinatorial approximation of the given boundary correspondence, to produce a triangulation of the sphere. We then compute the circle packing for 
this triangulation. For example, we may start with two maximal packings of the unit disc (meaning that the boundary circles are internally tangent to the unit circle), and use the arc length around the unit circle as a guide for pasting together the two triangulations, introducing new vertices where necessary to keep control of the vertex degrees.

In our application to intrinsic circle domains, we weld a combinatorial closed disc to the boundary cycle corresponding to each complementary component $B$ of $\Omega$, using the arc length around the circle corresponding to $v_{B}$ in the packing $\mathcal{P}_{B}$ and the trivial boundary correspondence $e^{i \theta} \mapsto e^{-i \theta}$. A suitable choice of combinatorial closed disc would be a large section of the regular hexagonal packing, then the hex packing lemma of Rodin and Sullivan can be applied to show that the desired welding and the computed welding differ by a quasiconformal map which has small dilatation except on an annulus of small modulus and small area covering the cycle of edges along which the welding is performed. After performing a welding for each boundary component, we simply apply Thurston's algorithm to compute a circle packing of the resulting spherical triangulation. In the case where the ambient Riemann surface $R$ has positive genus, we would compute a packing of the universal cover of the welded complex at this stage to get a circle packing approximation to the Riemann surface $S$.

The finished spherical circle packing gives an approximation to the map $\varphi$ and to the desired conformal structure on the interior of each complementary component of $\varphi(\Omega)$, and on the interior of the image of $\varphi(\Omega)$.

7.6. Convergence. We sketch a proof that the approximation scheme described above does converge locally uniformly on the domain $\Omega^{*}$ to the conformal mapping to an intrinsic circle domain, after suitable normalization. The strategy is to show that every map appearing in Figure 1 is well approximated by the corresponding polyhedral map between circle packings. Then since each step depends continuously on its input data, we will find that the polyhedral map from $\mathcal{P}^{*}$ to $S^{2}$ converges locally uniformly on $\Omega^{*}$ to the desired conformal mapping.

On the subcomplex of the packing representing the intrinsic circle domain, the hex-packing distortion estimate of Rodin and Sullivan shows that the polyhedral mapping is quasiconformal with dilatation that converges locally uniformly to zero. We can apply a similar estimate on the interior of each complementary component.

It remains to show that the conformal welding step is well approximated by the discrete conformal welding. To do this we have to control the conformal modulus of the image of a narrow annular neighborhood of each boundary component, where the circles are not deep enough in the hex packing for the Rodin-Sullivan estimate to give the bound we need. To do this it suffices to bound the maximum degree of the vertices appearing in the final triangulation. This is because the Rodin-Sullivan ring lemma allows the quasiconformal distortion of polyhedral maps between circle packings to be bounded in terms of the maximum degree. We can arrange that all vertices have degree 6 except those involved in the combinatorial welding. The largest degree arising when we weld using the hyperbolic radii of the neighbors of $v_{B}$ in the packing $\mathcal{P}_{B}$ can be bounded in terms of the ratio between the radii of the largest and smallest circles adjacent to $v_{B}$. Since we began with a hex-packing of $\Omega$ with circles all of equal radius, this ratio is controlled in the limit by the maximum and minimum value of the derivative on the boundary $\partial B$ of the conformal map $h \circ \pi^{-1}$. 


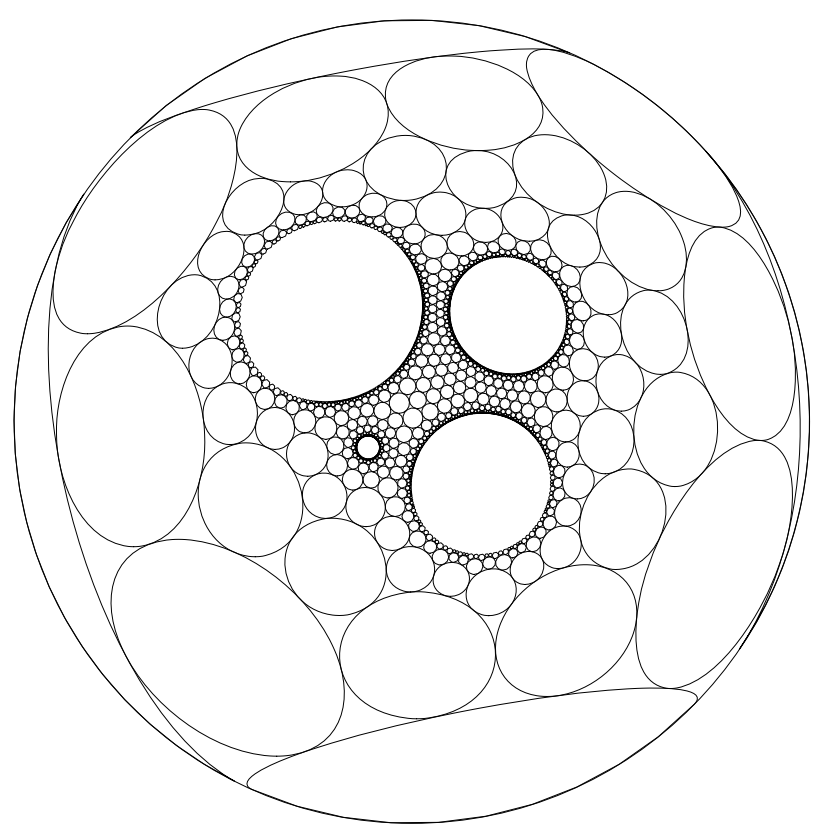

FiguRE 5. A circle packing of a circle domain with four complementary components.

7.7. Practical considerations. Examining the figures, the reader will note that in our numerical experiments we have not restricted ourselves to using hex packings as our starting point. Instead we have used packings with a few layers of degree 7 vertices near the boundary, so that there are many very small circles on the boundary without needing a very large number of circles in total. This was done to reduce the errors from the combinatorial welding step. We pay for this by having larger circles far from the boundary, and losing the rigorous distortion bounds, but this seems a worthwhile trade since we expect the Schwarzian derivative of the map that we are approximating to be small on this region. In Figures 3 and 4 there are circles of degree 15 fixed by a rotational symmetry of the packing of order 3 . The Schwarzian derivative of the map we are approximating must certainly vanish at the center of this circle, by symmetry considerations. Apart from two circles of this type, the maximum degree in the packing is 10. In the packings in Figures 5 and 6. the maximum degree is 7 .

Finally, we comment that it may be more satisfactory from the point of view of computation, and also to simplify the convergence proof, to perform the discrete conformal welding using packings with specified overlap angles rather than restricting ourselves to tangency packings. However, we have not implemented this in practice. 


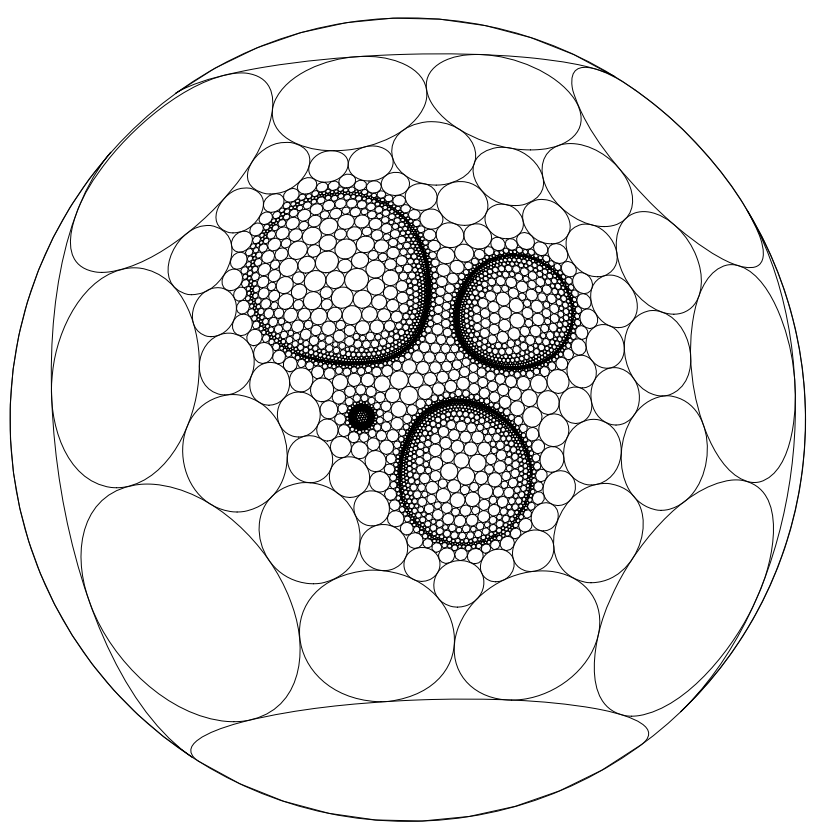

FiguRE 6. The spherical circle packing used to compute the intrinsic circle domain conformally equivalent to the circle domain shown in Fig. 5 .

\section{REFERENCES}

[1] John R. Akeroyd, Kristi Karber, and Alexander Yu. Solynin, Minimal kernels, quadrature identities and proportional harmonic measures, Rocky Mountain J. Math. 36 (2006), no. 6, 1819-1844. MR2305631

[2] Tom Carroll and Joaquim Ortega-Cerdà, The univalent Bloch-Landau constant, harmonic symmetry and conformal glueing, J. Math. Pures Appl. (9) 92 (2009), no. 4, 396-406, DOI 10.1016/j.matpur.2009.05.008 (English, with English and French summaries). MR2569185 (2010i:30009)

[3] Edward Crane, A bound for Smale's mean value conjecture for complex polynomials, Bull. Lond. Math. Soc. 39 (2007), no. 5, 781-791, DOI 10.1112/blms/bdm063. MR2365227 (2008i:30003)

[4] G. M. Goluzin, Geometric theory of functions of a complex variable, Translations of Mathematical Monographs, Vol. 26, American Mathematical Society, Providence, R.I., 1969. MR.0247039(40 \#308)

[5] Zheng-Xu He and Oded Schramm, Fixed points, Koebe uniformization and circle packings, Ann. of Math. (2) 137 (1993), no. 2, 369-406, DOI 10.2307/2946541. MR1207210(96b:30015)

[6] Joaquim Ortega-Cerdà and Bharti Pridhnani, The Pólya-Tchebotaröv problem, Harmonic analysis and partial differential equations, Contemp. Math., vol. 505, Amer. Math. Soc., Providence, RI, 2010, pp. 153-170, DOI 10.1090/conm/505/09921. MR2664566 (2011j:30032)

[7] Burt Rodin and Dennis Sullivan, The convergence of circle packings to the Riemann mapping, J. Differential Geom. 26 (1987), no. 2, 349-360. MR906396 (90c:30007)

[8] Oded Schramm, Transboundary extremal length, J. Anal. Math. 66 (1995), 307-329, DOI 10.1007/BF02788827. MR1370355 (96k:30009) 
[9] Alexander Yu. Solynin, Quadratic differentials and weighted graphs on compact surfaces, Analysis and Mathematical Physics, 473-505, Trends Math., Birkhäuser, Basel, 2009. MR2724628

[10] Kenneth Stephenson, Introduction to circle packing, Cambridge University Press, Cambridge, 2005. The theory of discrete analytic functions. MR2131318 (2006a:52022)

[11] K. Stephenson et al., CirclePack, software for circle packing, www. math.utk.edu/ kens/circlepack.

[12] G. Brock Williams, Discrete conformal welding, Indiana Univ. Math. J. 53 (2004), no. 3, 765-804, DOI 10.1512/iumj.2004.53.2392. MR2086700(2005f:30018)

Heilbronn Institute for Mathematical Research, School of Mathematics, University of Bristol, BS8 1TW, United Kingdom

E-mail address: edward.crane@bristol.ac.uk 